\title{
Scirrhous Adenocarcinoma
}

National Cancer Institute

\section{Source}

National Cancer Institute. Scirrhous Adenocarcinoma. NCI Thesaurus. Code C2928.

An infiltrating adenocarcinoma characterized by the presence of desmoplastic stromal reaction. 